\title{
Modelling near subsurface temperature with mixed type boundary condition for transient air temperature and vertical groundwater flow
}

\author{
Rajeev Ranjan Kumar ${ }^{1}$, D V Ramana ${ }^{2, *}$ and R N Singh ${ }^{2}$ \\ ${ }^{1}$ Department of Geology and Geophysics, Indian Institute of Technology, Kharagpur, 721902 India. \\ ${ }^{2}$ National Geophysical Research Institute, Hyderabad 500 00\%, India. \\ ${ }^{*}$ Corresponding author.e-mail: dvr@ngri.res.in
}

\begin{abstract}
Near-subsurface temperatures have signatures of climate change. Thermal models of subsurface have been constructed by prescribing time dependent Dirichlet type boundary condition wherein the temperature at the soil surface is prescribed and depth distribution of temperature is obtained. In this formulation it is not possible to include the relationship between air temperatures and the temperature of soil surface. However, if one uses a Robin type boundary condition, a transfer coefficient relates the air and soil surface temperatures which helps to determine both the temperature at the surface and at depth given near surface air temperatures. This coefficient is a function of meteorological conditions and is readily available. We have developed such a thermal model of near subsurface region which includes both heat conduction and advection due to groundwater flows and have presented numerical results for changes in the temperature-depth profiles for different values of transfer coefficient and groundwater flux. There are significant changes in temperature and depth profiles due to changes in the transfer coefficient and groundwater flux. The analytical model will find applications in the interpretation of the borehole geothermal data to extract both climate and groundwater flow signals.
\end{abstract}

\section{Introduction}

There is great interest to know past climate, especially to infer any anthropogenic signals in present climate by inter-comparison. The observations of subsurface thermal regime in boreholes have been used to infer earth's thermal history. However, these also can be used to find changes in the earth's surface temperature due to climate change. Lachenbruch and Marshall (1986) for the first time used borehole temperature data to infer the climatic signals which are due to time varying surface temperatures. Since then this field has extended interpreting all available global dataset using both forward and inverse methodologies to estimate the climate signals. Extensive literature has been reviewed by Bodri and Cermak (2007), Gonźalez-Rouco et al. (2009) and Davis et al. (2011). The methodology for interpreting borehole data involves using solutions of $1 \mathrm{D}$ heat conduction in half space with time dependent temperature boundary conditions. This solution is fitted with the borehole data to infer surface boundary condition discretized as step functions of time. Using geophysical inverse methodology, significant departures from the constant temperature boundary

Keywords. Borehole temperature; groundwater recharge rate; mixed type boundary condition; transient air temperature; climate. 
condition are found. Enhancement in surface temperatures since industrialization is also discernable in the borehole observations (Pollack and Huang 2000).

Borehole geothermal data also has signatures of other perturbations besides in surface temperatures, such as due to movement of groundwater (Anderson 2005; Constantz 2008; Swanson and Cardenas 2011). Thus the temperature-depth profiles are also used to estimate subsurface water fluxes because heat in subsurface is transported not only by conduction but also by heat advection caused by subsurface water flow. Effects of water flow on temperature have been studied by several researchers. Stallman (1963, 1965) derived the differential equation for combined conductive and groundwater advective heat transfer. Lubimova et al. (1963) considered effects of vertical flow of water in oceanic sediments. Bredehoeft and Papadopulos (1965) obtained analytical solution describing temperature profile with depth where fluid is moving vertically with constant velocity. Solutions for stratified media has also been derived by Negi and Singh (1967) and (Shan and Bodvarsson 2004). Reiter (2001) showed the estimation of horizontal and vertical groundwater flow components using precision temperature logs.

Kohl (1998) studied the effects of groundwater flows on the vertical geothermal profiles which are used for paleoclimate reconstructions. He obtained numerical flow in a 2D aquifer to show that climatic signals are not washed out by groundwater flows. Effects of permeability distribution on the nature of geotherms were investigated. He also applied on the temperature-depth profile inversion scheme using purely conductive forward model and noted differences in the GST results both in presence and absence of groundwater flow perturbations. He further developed a three dimensional model including joint effects of ground surface temperature (GST), topography and aquifer properties at KTB site in Germany. Ferguson et al. (2006) also studied synthetic borehole geothermal data perturbed by groundwater flow but used purely conductive models for inversion of climate signals. They were looking for likely magnitudes of groundwater flows which can have implication for construction of GST. Only high permeable, high recharge and deep circulating aquifers were found to be disturbing the reconstruction of surface temperature. In such cases it would be necessary to include changes in the groundwater recharge in GST reconstruction. Bodri and Cermak (2005) constructed synthetic borehole temperature profile using $1 \mathrm{D}$ advectiondiffusion equation and applied inversion methodology of 1D purely diffusion forward model to estimate effects of advection on GST construction. They showed that climatic signals would be distorted in presence of advection; climatic signals are not washed out due to convection. Reiter (2005) has jointly determined the groundwater flows and time varying ground surface temperatures by fitting analytical solutions with the observations. Taniguchi et al. (1999a, 1999b) presented a series of type curves for estimating vertical groundwater flux in sediment in which surface temperature increases linearly with time and groundwater flows vertically and applied to infer effects of urbanization and groundwater flows on subsurface temperatures. Taniguchi (2011) and Gunawardhana et al. (2011) have also studied the temperatures of groundwater to infer signals of urbanization and climate.

In above studies, the surface temperature has been prescribed to compute its effect on the subsurface temperatures. There have been some studies in simultaneous measurements and analysis of the GST and air surface temperatures (AST) at several sites, starting with Putnam and Chapman (1996). Beltrami and Kellman (2003) observed both air and ground surface temperatures at two sites and found no regular behaviour over long term between them, the offsets between them varying with time. Bartlett et al. (2006) and Davis et al. (2010) have looked at coupling between GST and SAT at decadal time scales and found strong coupling between them at these scales. Coupling of air and ground surface temperatures have also been analyzed by Stieglitz and Smerdon (2007). A general consensus in the literature is that GST derived from borehole data does mimic the surface temperature. However, with more simultaneous measurements of both GST and SAT, including in India (Akkiraju and Roy 2011a, 2011b), it would be interesting to prescribe AST and obtain subsurface temperatures to know climate and other signals in borehole data.

In this paper, we shall use a boundary condition at the earth's surface wherein a combination of heat flux and the surface temperature are prescribed in terms of surface air temperatures. This boundary condition is called Robin type boundary condition. The boundary condition wherein either temperature or heat flux is given in special cases of this general boundary condition. With this boundary condition, we construct an analytical model for transient air temperature at the surface and groundwater recharge in the subsurface. Quantitative studies are being done for different parameters like velocity of groundwater recharge and heat transfer coefficient. Generally GST inversion methodology uses 1D diffusive forward model with given surface temperatures. Several least squares and Bayesian inverse methods have been developed and used in the literature based on such a forward solution. Bodri and Cermak (2007) have 
summarized the literature, which can be referred for details. Our analytic solution can be used as forward model to develop inversion schemes to infer in addition to groundwater recharge.

\section{Mathematical formulation}

Transient temperature distribution in the subsurface in response to one-dimensional non-isothermal flow of an incompressible fluid through homogeneous porous media is governed by the following advection-diffusion equation:

$$
\begin{aligned}
\left(\frac{1}{\kappa}\right) \frac{\partial T}{\partial t} & =\frac{\partial^{2} T}{\partial Z^{2}}-\frac{U}{\kappa} \frac{\partial T}{\partial Z} \\
U & =\frac{\left(\rho c_{p}\right)_{f}}{\left(\rho c_{p}\right)_{s}} v_{f}
\end{aligned}
$$

where $T$ is the temperature, $Z$ is the depth from the surface (positive downwards), $t$ is time, $\kappa$ is the thermal diffusivity, $v_{f}$ is vertical (Darcy) fluid flow velocity (positive value for downward flow and negative value for upward flow), $\rho$ is the density and $c_{p}$ is specific heat. Subscript $f$ and $s$ refers to fluid and solid phases. The associated initial and boundary conditions are as follows:

$$
\begin{gathered}
T=T_{0}+a Z \text { at } t=0 \text { for all } Z>0 ; \\
K \frac{d T}{d Z}=H\left(T-T_{A}-b t\right) \quad \text { at } Z=0 \text { for all } t>0 .
\end{gathered}
$$

Equation (3) describes that the surface temperature is $T_{0}$ at time $t=0$ and initial temperature increase with depth with gradient given a constant a. Equation (4) is Robin type of boundary condition at the surface. Here coupling of the air temperature $\left(T_{A}\right)$ and soil surface temperatures $\left(T_{0}\right)$ are different, depending on the values of $H$, called the heat transfer coefficient. Values of $H$ depend upon winds and earth surface conditions. Such a boundary condition has been used in hydrothermal studies (Heasler et al. 1990). In case, the heat transfer coefficient $H$ tends to infinity, soil temperature $T_{0}$ at the surface $(Z=0)$ becomes the same as air temperature, and the boundary condition at the surface is then given by:

$$
T=T_{A}+b t .
$$

The constant, $b$, is the rate of increase (positive value of $b$ )/decrease (for negative value of $b$ ) of the surface air temperature. For generality, we have taken different initial soil temperature $\left(T_{0}\right)$ from initial air temperatures $\left(T_{A}\right)$. Thus we need the solution of equation (1) with initial condition as equation (3), surface boundary condition as equation (4) and basal boundary condition taken as finite value of temperature.

\section{Solution of the problem}

We will present our new results as case (3) and before that summarize two cases for which results are available in the literature. Our new result in case (3), generalizes these results.

Case 1: Given surface temperature $(T)$ with sudden change at $t=0$ and with linear increase at time $>0$ :

We use the method of Laplace transformation to solve the one-dimensional transient heat conduction equation (Carslaw and Jaeger 1959). Taking the Laplace transform of equation (1), using the initial condition equation (3), we get

$$
\frac{d^{2} \tilde{T}}{d Z^{2}}-\frac{U}{\kappa} \frac{d \tilde{T}}{d Z}-\frac{p}{\kappa} \tilde{T}=-\frac{T_{0}+a Z}{\kappa}
$$

where $\tilde{T}(Z, p)$ is the temperature in the Laplace domain.

$$
\tilde{T}(Z, p)=\int_{0}^{\alpha} T(Z, t) \exp (-p t) d t .
$$

Equation (6) is a second order ordinary differential equation. The surface boundary condition at $Z=0$, equation (5) is transformed as:

$$
\tilde{T}=\frac{T_{A}}{p}+\frac{b}{p^{2}} \quad \text { at } Z=0 .
$$

The solution of equation (6) using equation (8) is given by, ignoring the terms which goes to infinite at $Z$ tends to infinity,

$$
\begin{aligned}
\tilde{T}(Z, p)= & {\left[\frac{T_{0}+a Z}{p}\right]-\frac{a U}{p^{2}} } \\
& +\left[\frac{T_{A}-T_{0}}{p}+\frac{b+a U}{p^{2}}\right] \\
& \times \exp \left(\frac{U Z}{2 \kappa}-Z\left\{\frac{U^{2}}{4 \kappa^{2}}+\frac{p}{\kappa}\right\}^{1 / 2}\right) .
\end{aligned}
$$

To get the solution in the time domain we need to take an Inverse Laplace Transform of the 
equation (9). The final solution for the temperature is obtained as (Carslaw and Jaeger 1959):

$$
\begin{aligned}
T(Z, t)= & T_{o}+a Z-a U t+\frac{1}{2}\left(T_{A}-T_{0}\right) \\
\times & \operatorname{erfc}\left(\frac{Z-U t}{2 \sqrt{\kappa t}}\right)+\exp \left(\frac{U Z}{\kappa}\right) \\
& \left.\times \operatorname{erfc}\left(\frac{Z+U t}{2 \sqrt{\kappa t}}\right)\right\}+\frac{1}{2}(b+a U) \\
\times & \left\{(Z+U t) \exp \left(\frac{U Z}{\kappa}\right) \operatorname{erfc}\left(\frac{Z+U t}{2 \sqrt{\kappa t}}\right)\right. \\
& \left.\quad-(Z-U t) \operatorname{erfc}\left(\frac{Z-U t}{2 \sqrt{\kappa t}}\right)\right\} . \quad(10)
\end{aligned}
$$

This equation and its special cases have found several applications in geophysics.

Case 2: Given surface temperature with no sudden change at $t=0$ (equation 5 with $T(0)=T_{A}$ ):

If the initial air temperature is same as the surface temperature that means $T_{A}=T_{0}$, then the equation (8) reduces (Carslaw and Jaeger 1959)

$$
\begin{aligned}
T(Z, t)=T_{0}+ & a Z-a U t+\frac{1}{2}(b+a U) \\
\times\{ & (Z+U t) \exp \left(\frac{U Z}{\kappa}\right) \operatorname{erfc}\left(\frac{Z+U t}{2 \sqrt{\kappa t}}\right) \\
& \left.-(Z-U t) \operatorname{erfc}\left(\frac{Z-U t}{2 \sqrt{\kappa t}}\right)\right\} .
\end{aligned}
$$

This is the solution used by Taniguchi (2002) to estimate the past groundwater recharge rate in Tokyo and other areas.

Case 3: Mixed boundary condition at the soil surface (equation 4)

We take the Laplace transform of equation (4) to get:

$$
\frac{d \tilde{T}}{d Z}=K_{1}\left(\tilde{T}-\frac{T_{A}}{p}-\frac{b}{p^{2}}\right) \quad \text { at } Z=0 .
$$

The solution of the equation (6) associated with the boundary condition equation (12) is obtained in Laplace domain as:

$$
\begin{aligned}
\tilde{T}= & \frac{T_{0}+a Z}{p}-\frac{a U}{p^{2}} \\
& +\left[K_{1}\left(T_{0}-T_{A}\right)-a\right] \frac{\exp \left(m_{2} Z\right)}{p\left(m_{2}-K_{1}\right)} \\
& -\frac{K_{1}(a U+b)}{p^{2}\left(m_{2}-K_{1}\right)} \exp \left(m_{2} Z\right)
\end{aligned}
$$

where

$$
m_{2}=\frac{U}{2 \kappa}-\sqrt{\frac{U^{2}}{4 \kappa^{2}}+\frac{p}{\kappa}} .
$$

We have ignored the term which goes to infinity as $Z$ tends to infinity. To get the final solution for temperature in time domain, we need to take the Inverse Laplace transform for the equation (11). We obtain the analytical expression for temperature as:

$$
\begin{aligned}
& T(Z, t)=\left(T_{0}+a Z-a U t\right)+\frac{1}{2 K_{1}}\left\{K_{1}\left(T_{0}-T_{A}\right)-a+\frac{a U+b}{\kappa K_{1}}\right\} \\
& \left\{\begin{array}{l}
\left(\frac{2 K_{1} \kappa-U}{K_{1} \kappa-U}\right) \exp \left(K_{1} Z-K_{1} t\left(U-\kappa K_{1}\right)\right) \operatorname{erfc}\left(\frac{Z+\left(2 K_{1} \kappa-U\right) t}{2 \sqrt{\kappa t}}\right) \\
-\operatorname{erfc}\left(\frac{Z-U t}{2 \sqrt{\kappa t}}\right)-\frac{\kappa K_{1}}{\kappa K_{1}-U} \exp \left(\frac{U Z}{\kappa}\right) \operatorname{erfc}\left(\frac{Z+U t}{2 \sqrt{\kappa t}}\right)^{2}
\end{array}\right\} \\
& +\frac{a U+b}{2 U}\left\{(Z+U t) \exp \left(\frac{U Z}{\kappa}\right) \operatorname{erfc}\left(\frac{Z+U t}{2 \sqrt{\kappa t}}\right)-(Z-U t) \operatorname{erfc}\left(\frac{Z-U t}{2 \sqrt{\kappa t}}\right)\right\} \\
& -\frac{(a U+b) \sqrt{t}}{K_{1} \sqrt{\kappa}}\left\{\begin{array}{l}
\exp \left(\frac{U Z}{\kappa}\right)\left[\frac{1}{\sqrt{\pi}} \exp \left(-\left(\frac{Z+U t}{2 \sqrt{\kappa t}}\right)^{1 / 2}\right)-\frac{Z+U t}{2 \sqrt{\kappa t}} \operatorname{erfc}\left(\frac{Z+U t}{2 \sqrt{\kappa t}}\right)\right] \\
+\frac{1}{\sqrt{\pi}} \exp \left(-\left(\frac{Z-U t}{2 \sqrt{\kappa t}}\right)^{1 / 2}\right)-\frac{Z-U t}{2 \sqrt{\kappa t}} \operatorname{erfc}\left(\frac{Z-U t}{2 \sqrt{\kappa t}}\right)
\end{array}\right\}
\end{aligned}
$$

This is a more general analytical model of the subsurface advective-diffusive temperature distribution whose special solutions have been used for interpretation of the borehole data. The solution depends on several parameters such as $T_{0}, \alpha, T_{A}, b$,
$U, \kappa$ and $K_{1}$, their values, depth-time dependence of temperatures and heat flux. We shall now illustrate the changes in the temperature-depth distribution for changes in some controlling parameters of this solution. 
Table 1. Parameters: Symbols and values.

\begin{tabular}{ll}
\hline Parameters (symbols) & \multicolumn{1}{c}{ Values (units) } \\
\hline Time $(t)$ & $(\mathrm{s})(\mathrm{yr})$ \\
Depth from the surface (positive downwards) $(Z)$ & $(\mathrm{m})$ \\
Temperature $(T)$ & $\left({ }^{\circ} \mathrm{C}\right)$ \\
Temperature of soil surface at $t=0\left(T_{0}\right)$ & $15\left({ }^{\circ} \mathrm{C}\right)$ \\
Air temperature at $t=0\left(T_{A}\right)$ & $16.5\left({ }^{\circ} \mathrm{C}\right)$ \\
Thermal conductivity $(K)$ & $2.5\left(\mathrm{~W} / \mathrm{m}^{\circ} \mathrm{C}\right)$ \\
Thermal diffusivity $(\kappa)$ & $6.1 \mathrm{~m}^{2} / \mathrm{yr}$ \\
Vertical $\left(\right.$ Darcy) fluid flow velocity $\left(v_{f}\right)$ & $32,3(\mathrm{~m} / \mathrm{yr})$ \\
Density $(\rho)$ & \\
Specific heat $\left(c_{p}\right)$ & \\
Subscripts $(f, s)$ indicate fluid and solid & \\
Constant in mixed boundary condition $(H)$ & $0.05,0.1,0.2,0.5\left(\mathrm{~W} / \mathrm{m}^{2}{ }^{\circ} \mathrm{C}\right)$ \\
Temperature gradient with depth in the initial condition $(a)$ & $0.022\left({ }^{\circ} \mathrm{C} / \mathrm{m}\right)$ \\
Temperature gradient with time in surface temperature $(b)$ & $0,0.1,0.2\left({ }^{\circ} \mathrm{C} / \mathrm{Yr}\right)$ \\
$U$ & $\left(\rho c_{p}\right)_{f} /\left(\rho c_{p}\right)_{s} \nu_{f}$ \\
\hline
\end{tabular}

\subsection{Comparison of all three solutions}

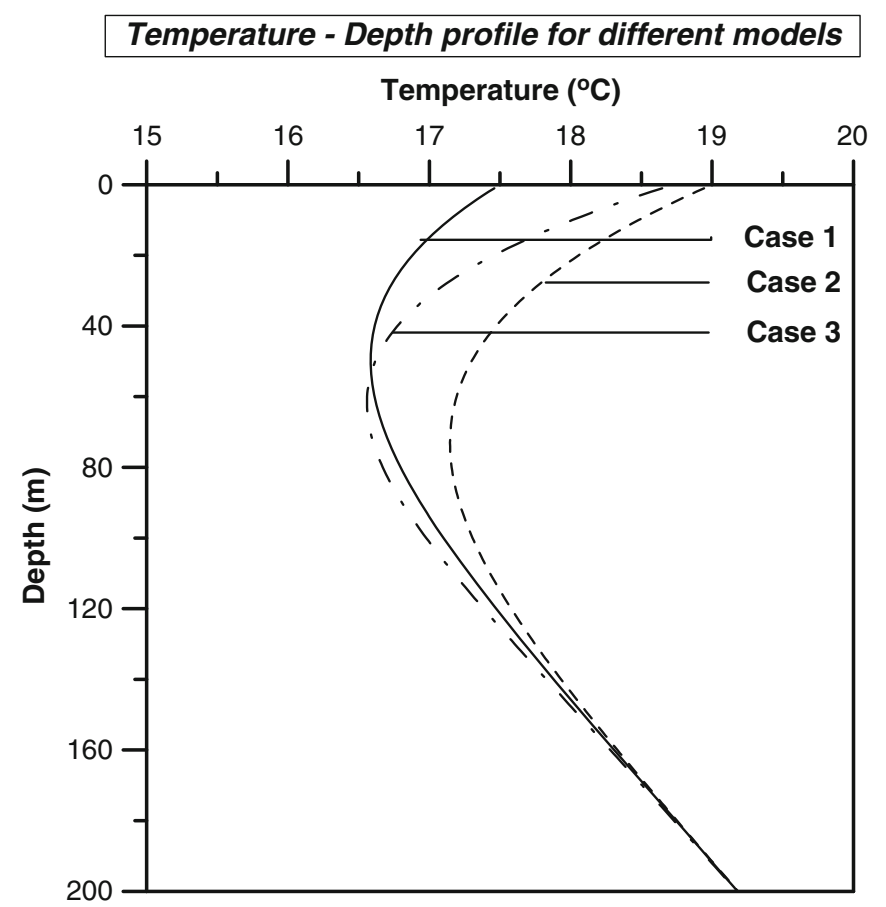

Figure 1. Calculated temperature-depth profiles based on different cases with $U=0.1 \mathrm{~m} /$ year, $b=0.01{ }^{\circ} \mathrm{C} / \mathrm{yr}$ and $H=0.2 \mathrm{~W} / \mathrm{m}^{2}{ }^{\circ} \mathrm{C}$ for $t=100 \mathrm{yrs}$.

\section{Numerical results and discussions}

We show the effects of changing values of heat transfer coefficient $(H)$, groundwater velocity $(U)$ and temperature gradient with time in surface temperature (b) using the equation (14). The numerical values taken for the calculations are given in table 1.
Figure 1 shows the temperature profiles for different surface boundary conditions. We have taken values of various parameters as: $U=0.1 \mathrm{~m} / \mathrm{yr}$, $b=0.01{ }^{\circ} \mathrm{C} / \mathrm{yr}, H=0.2 \mathrm{~W} / \mathrm{m}^{2}{ }^{\circ} \mathrm{C}$ and $t=100 \mathrm{yrs}$. Case 1, using equation (11) for a condition when the initial ground surface temperature $\left(15^{\circ} \mathrm{C}\right)$ is equal to the initial air temperature and also the surface temperature is a function of time (Taniguchi 2002). When the initial ground surface temperature is not equal to initial air temperature $\left(16.5^{\circ} \mathrm{C}\right)$ then the temperature profile has been calculated by using equation (8) and is plotted in figure 1 as case 2 . The temperature-depth profile has been calculated for mixed boundary condition (equation 12) and plotted as case 3. This figure shows that temperatures differ in near surface for all the cases but when it reaches to greater depths then the temperatures are same for all the cases.

\subsection{Effects of changes in temperature-depth profile with time}

To account for the effect of time on temperature with depth, the temperature-depth profile has been calculated at different times and is plotted in figure 2 for the values of $U, H$ and initial ground and air surface temperatures, same as in figure 1. This shows that till a depth of $80 \mathrm{~m}$ temperature increases with time and below this depth the differences from each other are small.

\subsection{Effects of changing rate of increase in air temperature}

The temperature-depth profiles have been calculated for different values of $b$, to see the effect of 


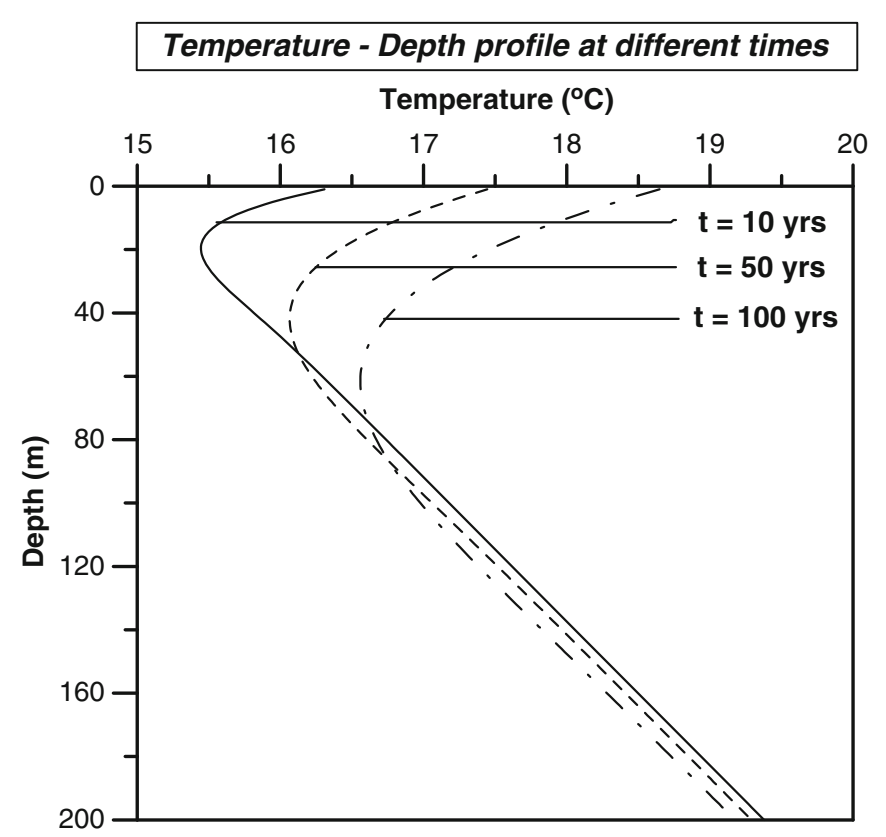

Figure 2. Calculated temperature-depth profiles based on parameter $t$ with $U=0.1 \mathrm{~m} / \mathrm{yr}$ and $H=0.2 \mathrm{~W} / \mathrm{m}^{2}{ }^{\circ} \mathrm{C}$.

Temperature - Depth profile for different values of $b$

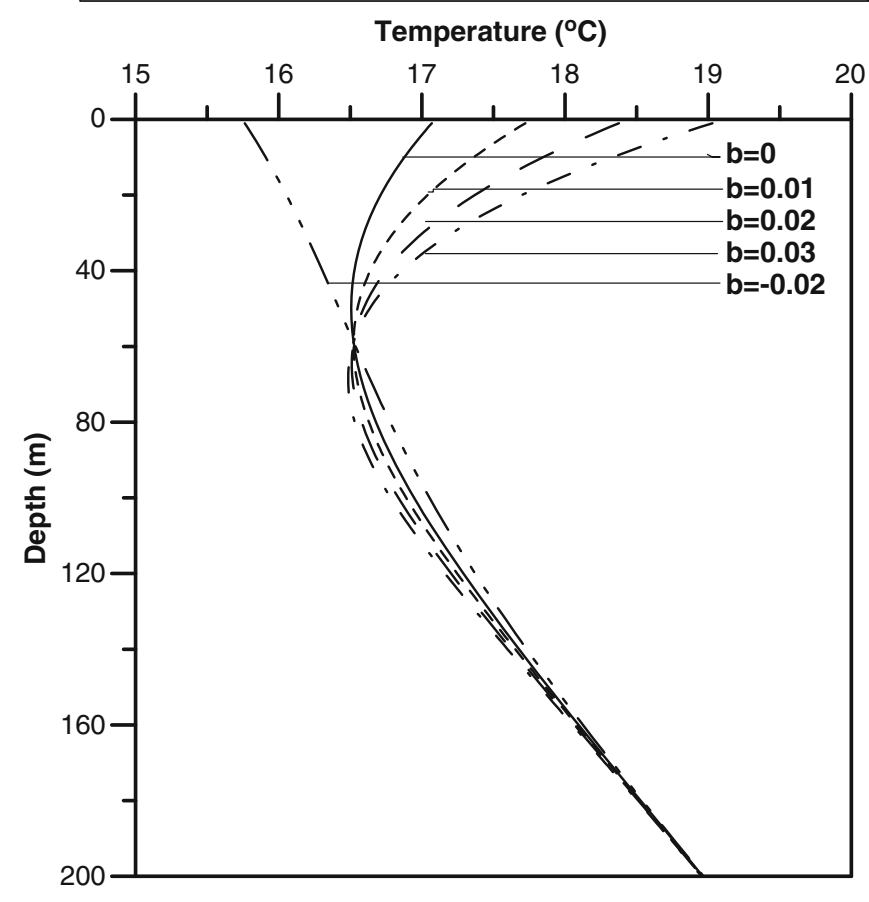

Figure 3. Calculated temperature-depth profiles based on parameter $b$ with $U=0.1 \mathrm{~m} / \mathrm{yr}$ and $H=0.2 \mathrm{~W} / \mathrm{m}^{2}{ }^{\circ} \mathrm{C}$.

time dependent surface boundary conditions. Figure 3 shows the plot of temperature with depth for different values of $b: b=0.0,0.01,0.02,0.03$ and $-0.02 \mathrm{C} / \mathrm{yr}$ at $100 \mathrm{yrs}$, all values of other parameters are same as in figure 2 . For all zero and positive values of $b$, the temperature decreases up to the depth of $50 \mathrm{~m}$ and then starts increasing. From
Temperature-Depth profile for different values of $U$

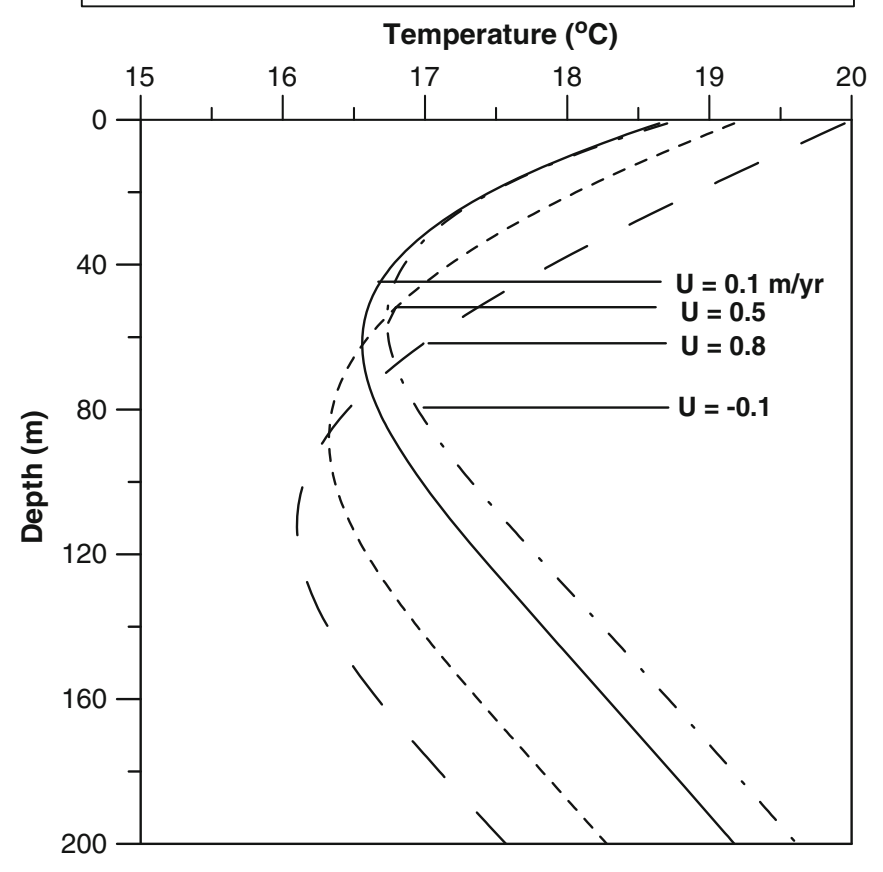

Figure 4. Calculated temperature-depth profiles based on parameter $U$ with $b=0.01{ }^{\circ} \mathrm{C} / \mathrm{yr}$ and $H=0.2 \mathrm{~W} / \mathrm{m}^{2}{ }^{\circ} \mathrm{C}$.

this figure it is evident that with higher values of $b$, there is a greater decrease in the temperature. We have also plotted the profile for a negative value of $b$. For this negative value of $b$ and downwards flux of groundwater, we find the soil surface temperature value decreases with time in contrast to the case of positive value of $b$, in which case soil surface temperature increases with time. Further, the temperature continuously increases with depth, in contrast to positive value of $b$ where the temperature decrease with depth initially till $50 \mathrm{~m}$ and then increases continuously with depth.

\subsection{Effects of changes in groundwater flux rate}

To account for effect of groundwater flux the temperature profile has been calculated for different values of vertical groundwater flux. The results have been shown in figure 4 for different values of $U$, i.e., $U=0.1,0.5$ and $0.8 \mathrm{~m} / \mathrm{yr}$, all values of other parameters are same as in figure 2 . The results show that the temperature decreases with increase in the rate of groundwater flux near the surface and then starts increasing with higher values of $U$. Greater the value of $U$, deeper the effect of temperature decline propagates downwards in the surface. Temperature profiles reverse after certain depth where effect of air and soil interaction minimizes. Near the surface top, temperature value increases with higher $U$, however at greater depths $(\sim 100 \mathrm{~m})$; temperature values will be lower for 
Temperature - Depth profile for different values of $\mathrm{H}$

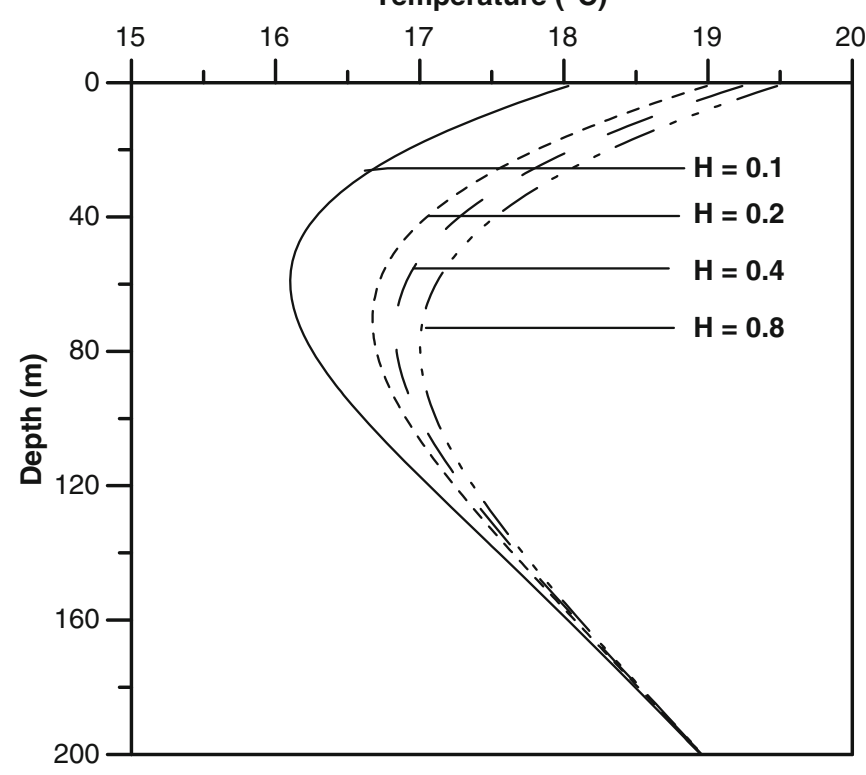

Figure 5. Calculated temperature-depth profiles based on parameter $H$ with $b=0.01{ }^{\circ} \mathrm{C} / \mathrm{yr}$ and $U=0.1 \mathrm{~m} /$ year.

higher value of $U$. Thus if the recharge rates are sufficiently high and the depth interval analyzed is sufficiently deeper, reasonably accurate values of $U$ can be estimated from actual temperature measurements. The GST history will be particularly crucial at shallower depths with lower $U$ values. We have also plotted temperature-depth profile for negative value of $U$, i.e., at $U=-0.1$. This case shows the effects of upward flow of groundwater, a case of discharge of groundwater. In this case the minimum value of the profile is larger than the profile for downward flow. Further the temperatures remain larger as depth increases than the case for downward flow. This is understandable as discharge of water from depths brings deeper heat towards the surface.

\subsection{Effects of changes in the heat transfer coefficient}

Heat transfer coefficient play important role in near surface heat transfer and temperature. To see the effect of heat transfer coefficient, depthtemperature profiles are calculated for various values of $H$ as shown in figure 5. The results show that the temperature profile varies with different $H$ values and temperatures are higher for higher values of $H$. After a certain value of $H$, differences are small and the temperature profiles coincide at greater depth. The effect of heat transfer coefficient is shown in figure 6 by comparing the air temperature and surface temperature variations with time for various values of $H$. Surface temperature

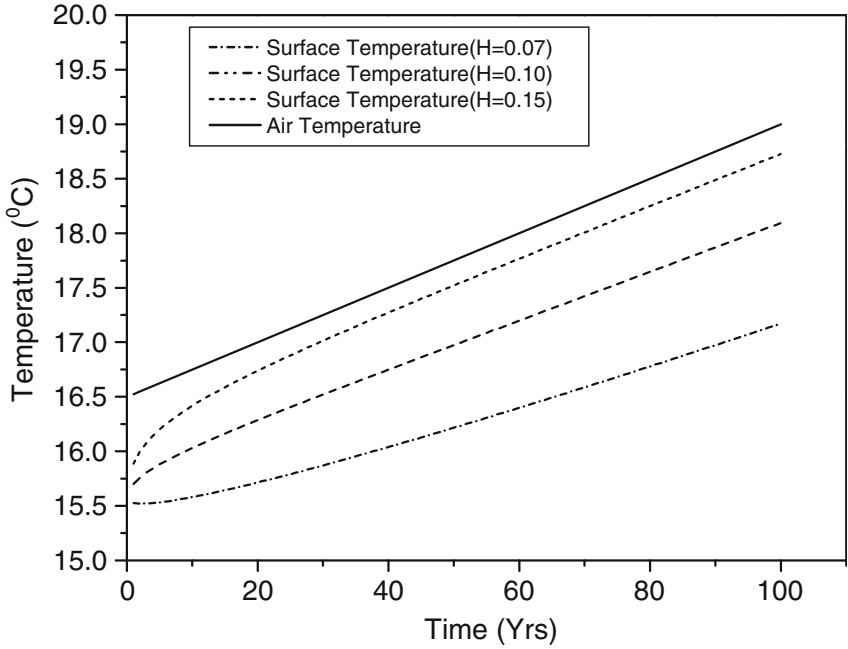

Figure 6. Calculated temperature-time profiles based on parameter $H$ with $b=0.025^{\circ} \mathrm{C} / \mathrm{yr}$ and $U=0.1 \mathrm{~m} /$ year.

follows the increase of the air temperature with time and the offsets between them are higher with lower values of $H$.

Thus there are significant changes in the temperature versus depth profiles for groundwater velocities, rate of increase/decrease in the air temperature, and heat transfer coefficients, which can be determined from the interpretation of observed subsurface, surface and air temperatures.

\section{Conclusions}

We have developed analytical model of the near subsurface temperature due to transient surface air temperature in the presence of groundwater vertical flows. We have used a Robin type boundary condition at the ground surface instead of Dirichlet type boundary condition which is usually used in interpretation of the borehole geothermal profiles for inferring climate signals. Numerical results have been presented for realistic thermal conditions to show the effects of variation in the heat transfer coefficient, groundwater velocities and rate of change of the air temperatures. As mentioned in the introduction, the inversion schemes for GST determinations have used mostly pure diffusive forward models. We plan to develop inversion schemes using this solution as a forward model to invert the geothermal data to infer both climate and groundwater flow signals.

\section{Acknowledgements}

The authors (RNS and DVR) are grateful to the Director, National Geophysical Research Institute for his kind permission to publish this work. RNS is thankful to INSA for award of a Senior 
Scientist position. $\mathrm{RR}$ is thankful to the Indian Academy of Sciences, Bangalore for providing IASc Summer Fellowship during 2006 when this work was carried out and Director, NGRI for providing supports to work at NGRI. Authors are also grateful to anonymous reviewer for very constructive and helpful suggestions.

\section{References}

Akkiraju V V and Roy S 2011a Geothermal Climate Change Observatory in south India 1: Borehole temperatures and inferred surface temperature histories; Physics and Chemistry of the Earth 36(16) 1419-1427.

Akkiraju V V and Roy S 2011b Geothermal Climate Change Observatory in south India 2: Set-up and first results; Physics and Chemistry of the Earth 36(16) 1428-1436.

Anderson M P 2005 Heat as groundwater tracer; Groundwater 43 951-968.

Bartlett, Marshall G, David S Chapman and Robert N Harris 2006 A decade of ground-air temperature tracking at Emigrant Pass Observatory, Utah; J. Climate 19 3722-3731, doi: 10.1175/JCLI3808.1.

Beltrami H and Kellman L 2003 An examination of shortand long-term air-ground temperature coupling; Global Planet. Change 38 291-303.

Bodri L and Cermak V 2005 Borehole temperatures, climate change and pre-observational surface air temperature mean: Allowances for hydraulic conditions; Global Planet. Change 45 265-276.

Bodri L and Cermak V 2007 Borehole climatology: A new method on how to construct climate; Elsevier, Amsterdam.

Bredehoeft J D and Papadopulos I S 1965 Rates of vertical ground water movement estimated from the Earth's thermal profile; Water Resour. Res. 1 325-328.

Carslaw H S and Jaeger J C 1959 Conduction of Heat in Solids, 2nd edn (New York: Oxford Univ. Press), 510p.

Constantz J 2008 Heat as a tracer to determine streambed water exchanges; Water Resour. Res. 44 W00D10, doi: 10.1029/2008WR006996.

Davis M G, Chapman D S and Harris R N 2011 Geothermal record of climate change, In: Encyclopedia of Solid Earth Geophysics (ed.) Gupta H K, Springer Science, doi: 10.1007/978-90-481-8702-7.

Davis M G, Harris R N and Chapman D H 2010 Repeat temperature measurements in boreholes from northwestern Utah link ground and air temperature changes at the decadal time scale; J. Geophys. Res. 115 B05203, doi: 10.1029/2009JB006875.

Gonźalez-Rouco J F, Beltrami H, Zorita E and Stevens M B 2009 Borehole climatology: A discussion based on contributions from climate modeling; Clim. Past 5 97-127.

Gunawardhana L N, Kazama S and Kawagoe S 2011 Impact of urbanization and climate change on aquifer thermal regimes; Water Resour. Manag. 25 3247-3276.

Ferguson, Grant, Hugo Beltrami and Allan D Woodbury 2006 Perturbation of ground surface temperature reconstructions by groundwater flow?; Geophys. Res. Lett. 33 L13708.

Heasler H P, George J H and Allen M B 1990 Improved computational schemes for the numerical modeling of hydrothermal resources in Wyoming: Final Report, U.S. Department of Energy.

Kohl T 1998 Paleoclimatic temperature signals - can they be washed out?; Tectonophys. 291 225-234.

Lachenbruch A H and Marshall B V 1986 Changing climate: Geothermal evidence from permafrost in the Alaskan Arctic; Science 234 689-696.

Lubimova I E A, Von Herzen R P and Udintsev G B 1963 On heat transfer through the ocean floor; In: Geophysical monograph series (ed.) Lee W H K, 8 78-86.

Negi J G and Singh R N 1967 On heat transfer in layered oceanic sediments; EPSL 2(4) 335-336.

Pollack H N and Huang S 2000 Climate reconstruction from subsurface temperatures; Ann. Rev. Earth Planet. Sci. 28 339-365.

Putnam S N and Chapman D S 1996 A geothermal climate change observatory: First year results from Emigrant Pass in northwest Utah; J. Geophys. Res. 101 21,877-21,890.

Reiter M 2001 Using precision temperature logs to estimate horizontal and vertical groundwater flow components; Water Resour. Res. 37(3) 663.

Reiter M 2005 Possible ambiguities in subsurface temperature logs - Consideration of groundwater flow and ground surface temperature changes; Pure Appl. Geophys. 162 $343-355$.

Shan C and Bodvarsson G 2004 An analytical solution for estimating percolation rate by fitting temperature profiles in the vadose zone; J. Contaminant Hydrol. 68 83-95.

Stallman R W 1963 Computation of ground water velocity from temperature data; U.S. Geol. Surv. Water Supply Pap., 1544-H, 36-46.

Stallman R W 1965 Steady one-dimensional fluid flow in a semi-infinite porous medium with sinusoidal surface temperature; J. Geophys. Res. 70 2821-2827.

Stieglitz M and Smerdon J E 2007 Characterizing landatmosphere coupling and the implications for subsurface thermodynamics; J. Climate 20 21-37; doi: 10.1175/JCLI3982.1.

Swanson T E and Cardenas M B 2011 Ex-stream: A MATLAB program for calculating fluid flux through sediment-water interfaces based on steady and transient temperature profiles; Comput. Geosci. 37 1664-1669.

Taniguchi M 2002 Estimation of the post groundwater recharge rate from deep borehole temperature data; Catena 48 39-51.

Taniguchi M 2011 Groundwater and subsurface environmental assessments under pressure of climate variability and human activities in Asia; In: Groundwater and subsurface environments: Human impacts in Asian coastal cities (ed.) Taniguchi M, Springer, doi: 101007/978-4-431-53904-9_1.

Taniguchi M, Shimada J, Tanala T, Kayane I, Sakura Y, Shimano Y, Dapaah-Siakwan S and Kawashima S 1999a Disturbances of temperature-depth profiles due to surface climate change and surface water flow: 1 . An effect of linear increase in surface temperature caused by global warming and urbanization in the Tokyo metropolitan area, Japan; Water Resour. Res. 35(5) 1507-1517.

Taniguchi M, Williamson D R and Peck A J 1999b Disturbances of temperature-depth profiles due to surface climate change and subsurface water flow: 2 . An effect of step increase in surface temperature caused by forest clearing in southwest Western Australia; Water. Resour. Res. 35(5) 1519-1529. 